\title{
Hobbes' Leviathan und die aus dem Blick gefallenen Schnabelmasken
}

\section{Francesca Falk}

Zusammenfassung: Dieser Artikel stellt das Frontispiz des Leviathan, das Emblem des politischen Körpers, in neue Zusammenhänge: und zwar in jene von Sanität, Souveränität und einer beginnenden Biopolitik. Die Schnabelmasken der in der Forschung kaum beachteten Pestärzte werden dabei zum Schwer- und Drehpunkt der Interpretation. Als Wahrnehmungsfilter verändern sie die Sicht auf den dargestellten Staatskörper.

Schlüsselwörter: Hobbes $\cdot$ Leviathan $\cdot$ Sanität $\cdot$ Souveränität $\cdot$ Selektion $\cdot$ Denkbilder des Politischen · Biopolitik

\section{Hobbes' Leviathan and the masks of the plague doctors that escaped critical attention}

\begin{abstract}
This article presents the frontispiece of the Leviathan, the emblem of the Body Politic, in new contexts. Starting from a detail mostly overlooked in previous analyses, the masks of the plague doctors, the author sketches a new picture of sovereignty, establishing a connection to sanitation and biopolitics. As the crux of his interpretation and as a kind of perceptual filter, the masks of the plague doctors alter the view of the represented body.
\end{abstract}

Keywords: Hobbes $\cdot$ Leviathan $\cdot$ Sanitation $\cdot$ Sovereignty $\cdot$ Selection $\cdot$ Body politic $\cdot$ Biopolitics

(C) VS Verlag für Sozialwissenschaften 2011

Dr. des. F. Falk $(\bowtie)$

Universität Basel und Zürcher Hochschule der Künste,

Lothringerstr. 79, 4056 Basel, Schweiz

E-Mail: Francesca.Falk@unibas.ch 
Das Frontispiz von Hobbes' Leviathan (1651) ist eine Ikone der politischen Philosophie geworden. ${ }^{1}$ Gerade in dieser Zeitschrift sorgt das imposante Bild seit Jahren für Diskussion (Bredekamp 2009, S.98; Manow 2007). Das Titelkupfer des Leviathan, das wahrscheinlich vom Pariser Künstler Abraham Bosse stammt (Bredekamp 2003), hat im Laufe der Geschichte zahlreiche Denkräume produziert - und ist zugleich ein Produkt derselben. Ein Bild, welches die Betrachter bannt und das politische Denken bahnt. Ein Bild aber auch, welches auf einigen Buchausgaben nicht zu finden ist (vgl. Hobbes 1966) und das erst im Zusammenwirken von Text und Bild zu einer Ikone wurde (Abb. 1).

Abb. 1: Abraham Bosse: Frontispiz des Leviathan, 1651, Britisch Library

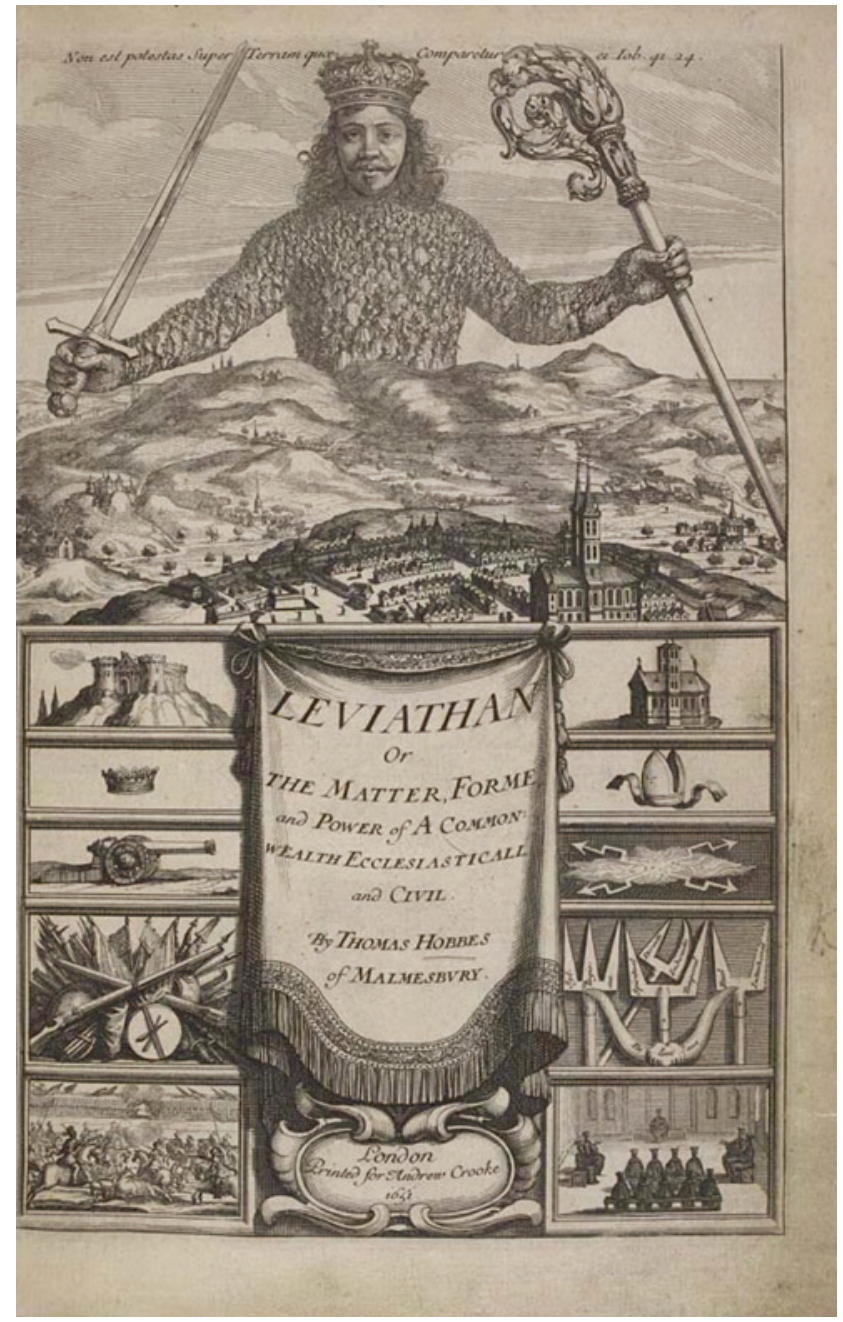

1 Dieser Artikel stützt sich auf ein Kapitel meiner noch unveröffentlichten Dissertation Falk 2011a. 
Abb. 2: Detailaufnahme Abb. 1

Abb. 3: Detailaufnahme Frontispiz des Leviathan, 1750, Britisch Library
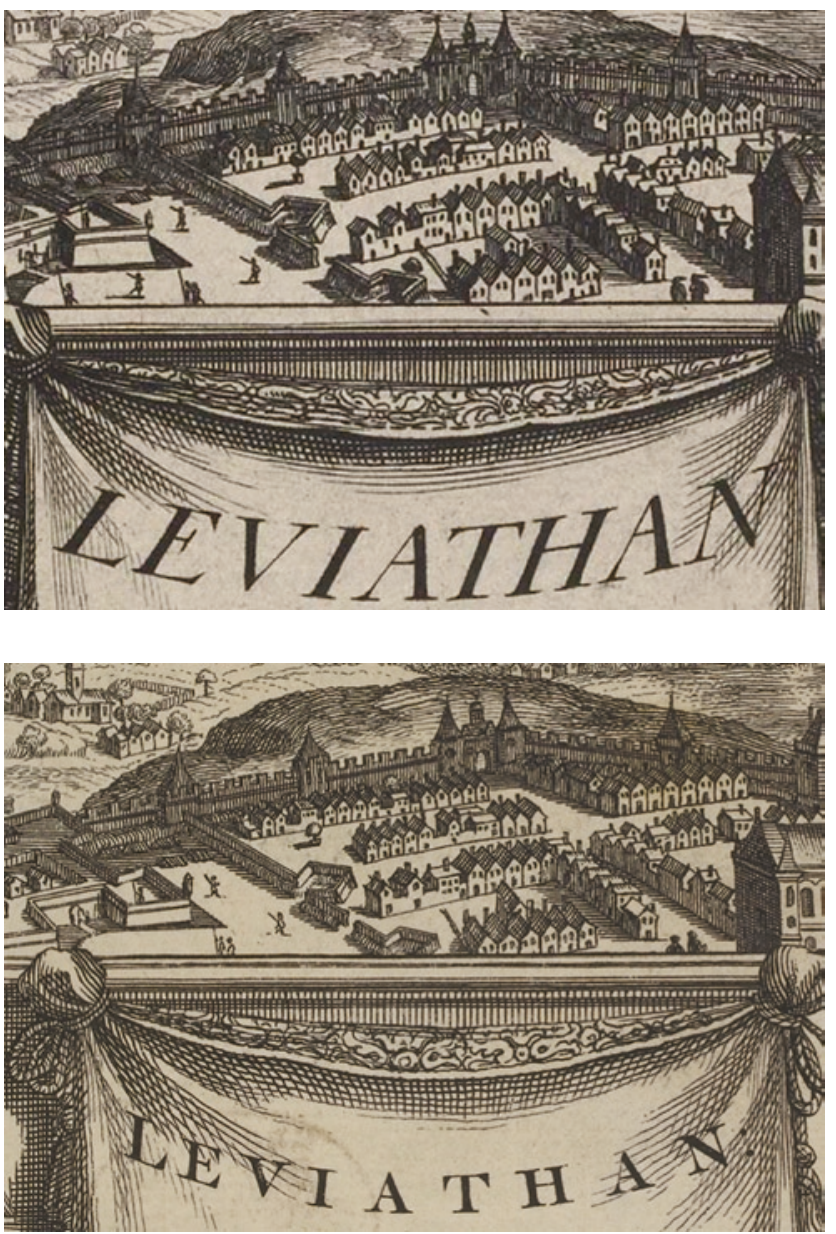

Das Frontispiz führt uns die Beziehung zwischen Schutz und Gehorsam vor Augen: Als Gegenleistung für Gehorsamkeit liefert der Leviathan Schutz. Dieser Schutz ist indes nicht nur militärisch gedacht: Er weist noch eine weitere Dimension auf. Damit wir diese sehen können, müssen wir uns näher ans Bild heranwagen (Abb.2 und 3).

Hier interessieren die beiden Figuren der Seuchenärzte. Deren Schnabelmasken sind zumindest zeitweise im wörtlichen wie metaphorischen Sinn aus dem Bild gefallen. Im holländischen Frontispiz von 1667 und in der Ausgabe von $1750^{2}$ beispielsweise finden wir als Gestalten vor der Kirche nicht mehr Seuchenärzte, sondern Besucher. ${ }^{3}$ Die Schnabelmasken tauchen in jenen Ausgaben, auf denen sie abgebildet sind, als unscheinbares Detail auf, als etwas, das da, aber kaum sichtbar ist, sich dem Blick entzieht und erst jenen

2 Diese wurde allerdings auch mit der Jahreszahl 1651 beschriftet.

$3 \mathrm{Im}$ Folgenden werde ich nicht danach fragen, weshalb diese Seuchenärzte in einigen Ausgaben nicht vorhanden sind; dieser Frage nachzugehen, wäre ein lohnendes Unterfangen, kann hier aber nicht geleistet werden. 
Betrachtern auffällt, die das Bild sezieren. Die Seuchenärzte befinden sich am äußersten Rand des oberen Bildabschnittes, auf der Grenze des gerade noch Sichtbaren und über jenem Vorhang stehend, der nach Carl Schmitt andeutet, „dass hier nicht nur viel gesagt, sondern ausserdem auch einiges verborgen ist" (2003, S. 151). Schmitt fügte jedoch seiner von antisemitischen Untertönen nicht freien Publikation von 1938 das erwähnte Frontispiz von 1750 bei; hier fehlen die Seuchenärzte (vgl. Schmitt 1938, S. 27; Bredekamp 2009). Die Schnabelmasken sind nicht nur aus dem Bild herausgefallen, sondern auch aus den Bildanalysen. Horst Bredekamp beispielsweise erwähnt die Schnabelmasken, entwickelt aber daraus kein eigenes Argument (2003, S. 27). Reinhard Brandt wiederum hat die in der Stadt dargestellten Menschen als Bürger interpretiert. Er schreibt: ,historisch pointiert könnte man von einer Zäsur zwischen Bourgeois und Citoyen sprechen: es werden die Menschen in ihrer privat-gesellschaftlichen Tätigkeit von der Person im Staat getrennt" (2000, S.318). Die staatliche Funktion der Seuchenärzte sowie die Schnabelmasken fallen hier aus dem Blick. Es wäre weiter interessant, systematisch der Frage nachzugehen, wo und wie Hobbes sich in anderen Schriften zu Seuchen und insbesondere der Pest äußert. Bei seiner Übersetzung des Thukydides sind Seuchen jedenfalls ein Thema. Von Bedeutung ist hier, dass Thukydides die Folgen der Pest als „anomia“, das heißt Gesetzlosigkeit schilderte, was an die Beschreibung des Naturzustands erinnert (Ginzburg 2008; Leven 2001; Orwin 1988). Carlo Ginzburg, der auf diesen Zusammenhang in einem aufschlussreichen Artikel aufmerksam macht, erwähnt ebenfalls nicht die Schnabelmasken, die sein Argument stützen würden.

Erstaunlich ist jedoch, dass ein so winziges Detail wie die beiden millimetergrossen Schnabelmasken auf dem Frontispiz von 1651 bei genauem Hinsehen klar erkennbar ist. Die Schnabelmaske besitzt eine sehr auffällige Form, ja sie ist im Laufe der Geschichte selbst gewissermassen zur Ikone geworden. Sie gehört zu der eigentümlichen Berufskleidung der Pestdoktoren. Schauen wir uns deren Entstehung etwas genauer an.

\section{Das ikonische Kostüm des Pestarztes}

Der Anzug des Seuchenarztes bestand aus einem Mantel aus luftundurchlässigem Material, Schutzhandschuhen, einer Kopfbedeckung und einer Maske mit Augenschutz und schnabelartiger Nase, ${ }^{4}$ die manchmal vor der Pest schützen sollende Räucherstoffe enthielt (vgl. Ulbricht 2005); diese Duftstoffe konnten tatsächlich auch Flöhe fernhalten. 1651 - als das Frontispiz entstand - war diese Form der Kleidung noch recht neu. Der spätere Leibarzt von Ludwig dem XIV, Charles de l'Orme, soll sich 1619 beim Ausbruch der Pest in Paris als einer der ersten ein solches Pestkleid angefertigt haben (vgl. Broquet 1911, S. 11; Brossollet 1979, S. 64). Dieses Kleid kennt jedoch viele Vorformen, wobei sich die dahinter stehenden Ansteckungs- und Miasmentheorien (Siegert 2006, S. 120-121) nicht scharf voneinander trennen lassen. Die Schnabelmaske beispielsweise verweist auf die Angst, dass die Pest über die Luft übertragen wie durch giftige Dämpfe ausgelöst werden könne. Offenbar hat sich das Pestkleid von Frankreich aus erfolgreich

4 Vielleicht steht die Schnabelform auch in einem Zusammenhang mit dem Umstand, dass Vögeln Immunität gegen die Pest zugeschrieben wurde. 
in andere Länder ausgebreitet (vgl. Salzmann 1932, S. 11). Es weist weiter eine apotropäische Dimension auf; die Schnabelmaske soll die Pest selbst erschrecken. Das Kleid des Seuchenarztes bewirkt zwei Dinge: Es macht die Funktion des Pestdoktors sichtbar und gleichzeitig seine Person unerkennbar. Der Pestarzt ist in seiner meist dunklen Montur und mit dem weißen Stock von weitem identifizierbar, und sein Gesicht bleibt hinter der Maske geschützt (Abb.4).

Eine der zumindest im deutschsprachigen Raum bekanntesten Pestarztdarstellung ist heute der Kupferstich Der Doctor Schnabel von Rom (1656) des Kunsthändlers und Verlegers Paulus Fürst (nach I. Columbina). Akzentuiert wird die Schnabelmaske durch die Worttrennung im Titel. Die beflügelte Sanduhr verkörpert Vergänglichkeit: tempus fugit. Die deutsch-lateinischen Verse erzeugen eine satirische Wirkung; der Pestdoktor erscheint hier vor allem als geldgieriger Kinderschreck. Pestärzte gingen ein hohes Risiko ein, weshalb nicht nur gutes Geld (wie in den Versen evoziert wird), sondern des öfteren auch ein freies Bürgerrecht lockte (vgl. Ulbricht 2005, S. 110). Der Doctor Schnabel von Rom ist einige Jahre nach dem Frontispiz entstanden. Interessanterweise tauchen Schnabelmasken auch im Karneval auf (Abb. 5). ${ }^{5}$

So auch in Johannes Lingelbachs Ölbild Carneval in Rom (Boeckl 2000), das vom Kunsthistorischen Museum Wien auf die Jahre 1650/1651 datiert wird, also in etwa auf die gleiche Entstehungszeit wie das Hobbessche Frontispiz. Auffallend sind hier die dichte Menschenmasse und die dennoch plastisch und präzise gestalteten Figuren (Levine und Ekkehard 1991). Am linken Rand sehen wir einen berittenen kostümierten Pestarzt und ein weiterer, stehend, unmittelbar davor. Beide fallen allerdings in der Menge kaum auf, wäre da nicht die Zeigegeste eines Reiters und eines ins Bild ragenden Arms mit ausgestrecktem Zeigefinger. Im Vordergrund sehen wir einen betrunkenen Schweizergardisten, der sich von der Menge löst und den Bildbetrachtern entgegen wankt; er entfernt sich dabei vom Dottore, der uns den Rücken zukehrt. Im Gegensatz zum Dottore, der sich auf seinem Pferd und mit seinem dicken Buch und breitem schwarzen Hut gut sichtbar im Vordergrund des Bildes befindet, stehen die beiden hier hellgekleideten Vogelfiguren wie beim Leviathan am Rand. Der Dottore zeigt mit ausgestrecktem Arm und einer Narren-

5 Ähnliche Schnabelmasken wie jene des Doctors von Rom werden heute als Souvenir in Venedig verkauft. Im venezianischen Karneval waren auch Judennasen ein beliebtes Motiv. Bekanntlich wurden Juden, die in Venedig im Ghetto lebten, immer wieder für Pestausbrüche verantwortlich gemacht. Weist die Schnabelmaske des Pestarztes, die ja die Krankheitsübertragung verhindern soll, auch einen Bezug zum Klischee der früher erscheinenden (tropfenden) Judennase auf, die spätestens ab dem 13. Jahrhundert auftaucht? Prominente Nasenformen sind allerdings auch bei anderen Masken zu beobachten, zudem bezeugt eine visuelle Verwandtschaft nicht zwingend eine geteilte Geschichte. So ist äußerliche Ähnlichkeit einer japanischen Pestuniform von 1910 und einer Schnabelmaske aus einem venezianischen Lazarett aus dem 14. Jahrhundert insofern auch irreführend, als das, was dazwischen liegt, dabei vielleicht zu leicht vergessen wird, so beispielsweise die entdeckte Ausbreitung über den Rattenfloh und die Isolierung des Pesterregers, was während der Pestpandemie der 1890er Jahre möglich wurde. Ein wesentlicher Unterschied besteht schliesslich weiter darin, dass sich in der Maske von 1910 der symbolbeladene Schnabel nicht mehr findet. 


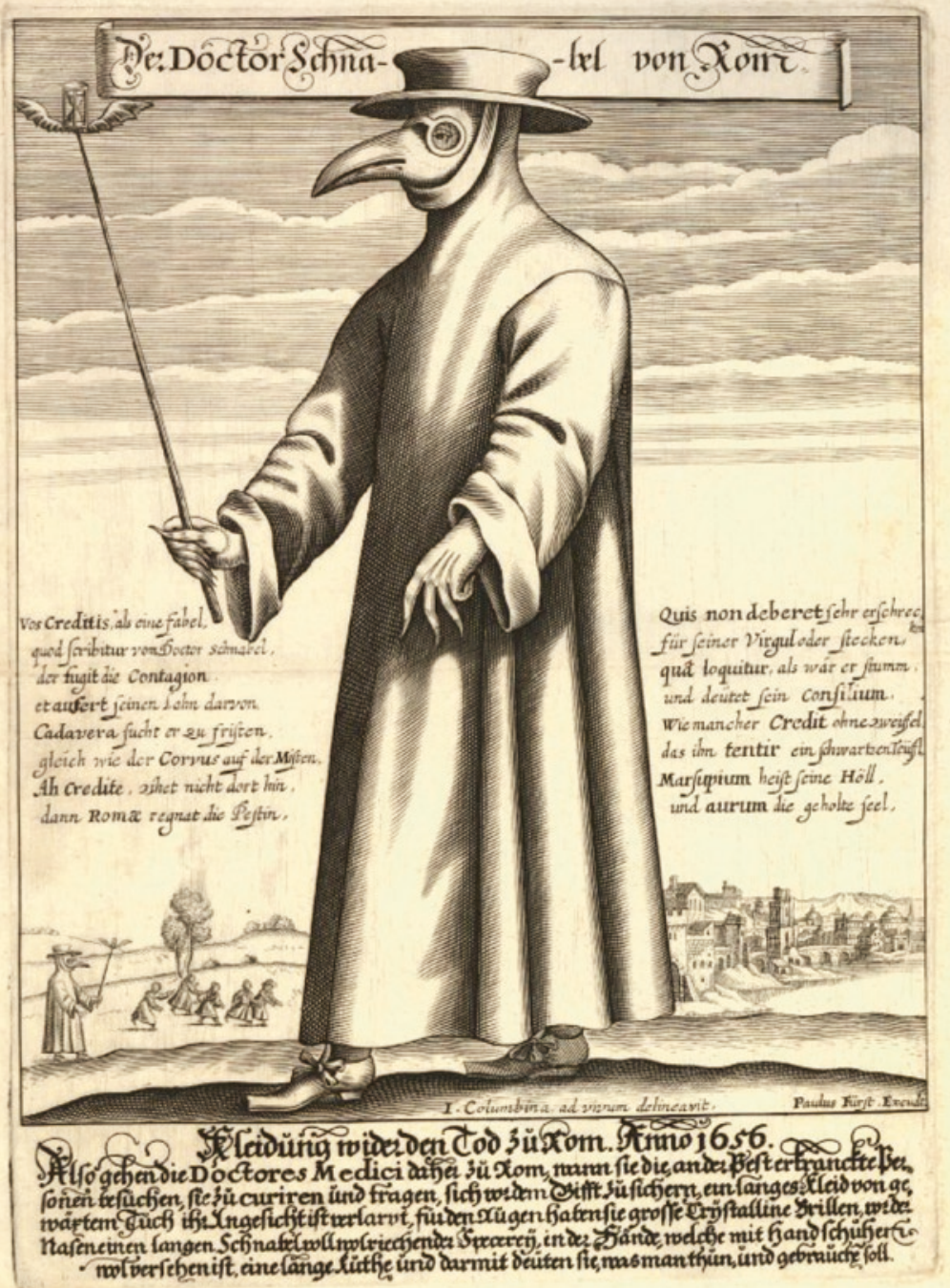

Abb. 4: Paulus Fürst nach I. Columbina: Der Doctor Schnabel von Rom, 1656. Goldhahn, Richard. 1940. Spital und Arzt von einst bis jetzt, 103. Stuttgart: Ferdinand Enke Verlag S. 103

kappe in der Hand auf einen Narrenkäfig. ${ }^{6}$ Die Einsperrung in Narrenkäfige diente dabei, ähnlich wie das Spektakel des Karnevals, der Unterhaltung der Stadtbewohner. Als Nar-

6 Zur Figur des Narren arbeitet Maike Christadler in ihrem Projekt „Figuren des Medialen - Formierungen des Imaginären. Exemplarische Analysen des Bilddiskurses um 1500“. 


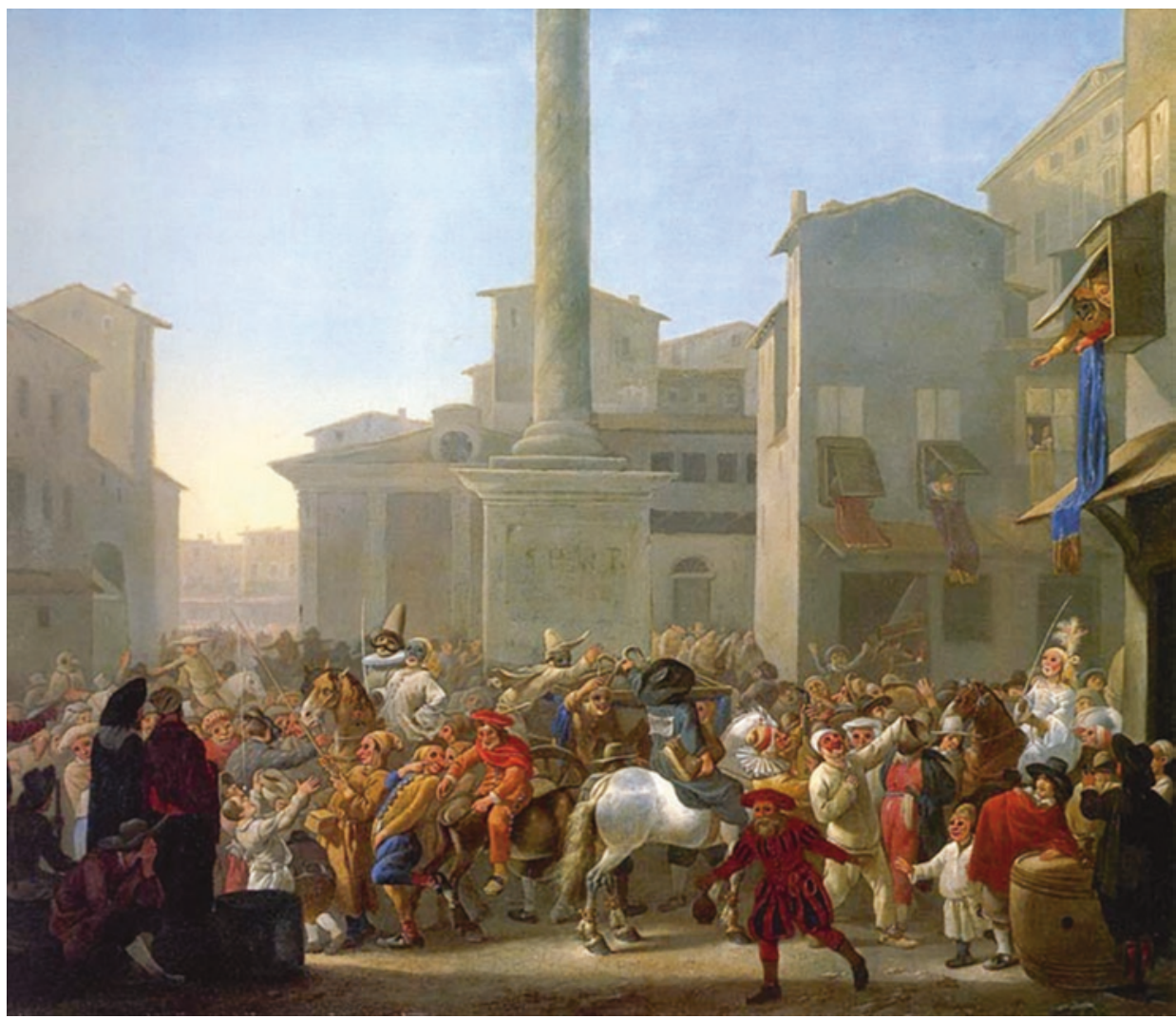

Abb. 5: Johannes Lingelbach: Carneval in Rom, wahrscheinlich 1650/1651, Kunsthistorisches Museum Wien

renhäuser wurden in Mitteleuropa denn auch häufig leer stehende ehemalige Leprosorien und Pesthäuser genutzt (Noack 2009).

In Zeiten des „Ausnahmezustandes“ wurde eine Verhüllung als Gefahr wahrgenommen (vgl. von Müller 1984, S. 126-132). So wurde in Italien im 16. Jahrhundert das Tragen von Karnevalsmasken in Zeiten der Pest mit dem Argument kritisiert, dadurch könne sich der Kranke ungesehen unter die Gesunden mischen (vgl. Bazin 1971, S. 76). Beide, (Toten-)Maske und Arzt, können als Memento mori fungieren. Die Starrheit der Maske veranschaulicht den Tod; Fastnachtsmasken waren weiter ursprünglich auch als Verbildlichung von Ahnenseelen gemeint (Weihe 2004, S. 22). Wie Masken einen Kontakt zwischen dem Lebenden und dem Toten herstellen, ,pendelt“ der Pestarzt zwischen beiden. Der Karneval dient der Repräsentation einer Gesellschaft in ihren Grenzen; in der ritualisierten Ausgelassenheit und Entgrenzung wird die gesellschaftliche Ordnung scheinbar auf den Kopf gestellt und dennoch gleichzeitig bekräftigt. Der Karneval selbst kann als ein lebendiges, ephemeres Bild der ,begrenzten Entgrenzung“ begriffen werden.

7 Bereits die griechische Theatermaske sollte zeigen, dass der Ausdruck der Theaterfigur vom Ausdruck des Schauspielers unabhängig gemacht werden kann (Weihe 2004). Dies entspricht in gewisser Weise der Differenz zwischen Amt und Amtsinhaber beziehungsweise Funktion und 


\section{Souveränität und Sanität}

Das Frontispiz betont die kompakte Ganzheit und Abgeschlossenheit des Staatskörpers, denn ,nur im Bild und nirgendwo sonst kann das Ganze des modernen Staates unmittelbar zur Anschauung kommen“ (König 2008, S.328). Die Schnabelmasken unterlaufen aber diese bildliche Suggestion einer totalen Inklusion. Sie erinnern an Selektionen und Exklusionen und sie bringen den Zusammenhang von Seuche, Sanität und Souveränität ins Bild.

Mit der Pestpolitik etablierten sich neue städtische bzw. staatliche Strukturen (Dinges 1995). George Rosen postuliert eine Parallele zwischen Public-Health-Maßnahmen und „the great Leviathan, the modern state, whose outlines slowly appear out of the storm sea of politics like a whale coming to the surface“" (Rosen 1958, S. 109). Die Stadt diente dabei als Vorbild für den Staat (vgl. Foucault 2004a, S. 483). Dabei entstanden neue Formen von Grenzkontrollen: Erinnert sei hier an das Aufkommen von Quarantänemaßnahmen ab dem 14. Jahrhundert, welche jedoch nicht eine hermetische Abriegelung zum Ziel hatten, sondern vielmehr als Membrane wirken sollten, die den Handel nicht blockieren, sondern sicher zu ermöglichen hatten. Auch die Entstehung des Passes lässt sich u. a. auf die in Pestzeiten ab dem 15. Jahrhundert ausgestellten „,bollettini di sanità “ zurückführen (Bohn 2006; Groebner 2002). Der Pestbrief diente als Grundlage für die Entscheidung, ob ein Reisender in die Stadt eingelassen wurde - oder eben nicht. Neben diesen Identifikationstechnologien etablierten sich neue Formen der Bewegungskontrolle. So wurde beispielsweise im Venedig des 15. Jahrhunderts die „Migration“ aus dem heutigen Balkan mit der Begründung der Pestprävention verboten (vgl. Palmer 1978, S. 54). Auch Bettler wurden seit 1526 regelmäßig aus der Stadt vertrieben (vgl. Palmer 1978, S. 215). Disziplinierungs- und Kontrollmassnahmen betrafen - allerdings in etwas anderen Dimensionen - auch die Bürger der Städte. Besondere Rechte wurden in Venedig den Provveditori alla Sanità beispielsweise in Bezug auf ihre Untersuchungsmethoden zugestanden. So wurde ihnen zu Beginn des 16. Jahrhunderts gestattet, Folter einzusetzen, um die Wahrheit über die Ursache von Seuchenausbrüchen aufzudecken (vgl. Palmer 1978, S. 84; Prantl 2008, S. 54-58). In diesem seuchenpolitischen Ausnahmezustand (vgl. Agamben 2004) gewannen demnach städtische Strukturen an Stärke; es ist kein Zufall, dass die Institution der Polizei in der frühneuzeitlichen „Gesundheitspolizey“ u. a. ihre Anfänge findet (vgl. Foucault 2004a, S. 451-481).

Doch Verordnungen garantieren noch nicht deren Umsetzung. Während die italienischen Stadtstaaten bezüglich ihrer Seuchenpolitik eine Vorreiterrolle einnahmen, wurden in Frankreich nach Richard Palmer solche Strukturen erst ab Mitte des 17. Jahrhunderts dauerhaft etabliert, in England noch später: „In 1626 a former ambassador to Venice, Sir Dudley Carleton, argued in the English Parliament for a bill on plague control, pointing out the success of plague measures in Paris and Venice" (Palmer 1978, S. 148). Seuchenpolitik erwies sich als wichtig für die Ausbildung von Souveränität, für die Kontrolle von

Person, wie sie Ernst Kantorowicz in seiner klassischen Studie zu den zwei Körpern des Königs formulierte vgl. Kantorowicz 1992. In diesem Zusammenhang ist der Begriff der Person, der ja unter anderem auf die Maske des Schauspielers (persona) zurückgeführt wird, insofern beachtenswert, als Hobbes den Staat als ,persona ficta“ denkt. 
innen und aussen. Die Provveditori alla Sanità konnten in Pestzeiten verbieten, Messen abzuhalten, denn die Zusammenkunft von Menschenmassen bedeutete eine Ansteckungsgefahr (vgl. Palmer 1978, S. 300). Auch nach Hobbes gehört es zu den Rechten des Souveräns, religiöse Ansammlungen zu verbieten (vgl. Hobbes 1966, S. 357). Er vergleicht unrechtmäßige Menschenversammlungen mit Geschwüren, die durch Zusammenfluss übler Säfte erzeugt werden. Hobbes greift vielfach auf eine medizinische Metaphorik zurück. Bereits in der Einleitung schreibt er, Eintracht im Staat sei Gesundheit, Aufruhr führe zu Krankheit, Bürgerkrieg aber bedeute den Tod des Staates (vgl. Hobbes 1966, S. 5).

Explizit von Seuchen spricht Hobbes im Kapitel „Von Wundern, und wozu sie bewirkt werden“. So habe Moses seine Hand aussätzig gemacht und anschließend wieder geheilt, um die Israeliten von seiner Auserwähltheit und Macht zu überzeugen; dies erinnert auch an die Stelle aus dem Buch Hiob (vgl. Hobbes 1966, S. 336). ${ }^{8}$ Gott habe eine Seuche über Ägypten geschickt, um den Pharao zu zwingen, die Israeliten ziehen zu lassen. Dieser Eingriff Gottes hatte laut Hobbes indes nicht zum Ziel, den Pharao zu bekehren, denn der Kirche sollten nicht alle Menschen zugeführt werden, sondern nur jene, die erlöst werden sollten, das auserwählte Volk. Hier wird die Seuche ebenfalls in eine enge Verbindung mit Selektionsprozessen gebracht; die magische Heilung der aussätzigen Hand richtet sich als Signal nur an Auserwählte. Es sollte indes auch im Auge behalten werden, dass Hobbes im Leviathan zwar auf Seuchen, nie aber auf eine eigentliche Seuchenpolitik zu sprechen kommt.

\section{Bevölkerung}

Im Zusammenhang mit der Seuchenabwehr entdeckte die städtische Gewalt den Nutzen der Statistik (vgl. Fazzini 2004; Brusatin 1981). Ein wichtiger Vordenker der Bevölkerungsstatistik war William Petty. Dieser hatte eine politische Arithmetik entworfen, in der die Bevölkerung, so Achim Landwehr, nicht als gegeben, sondern als gemacht konzeptualisiert worden sei (vgl. Landwehr 2007, S. 292). Petty, der in Holland medizinische Vorlesungen besucht hatte, kam 1645 nach Paris. Hier traf er Hobbes - der zwischen 1640 und 1651 im Pariser Exil war - und wurde dessen Assistent (vgl. Jordan 2007, S. 35). Petty fertigte Zeichnungen für Hobbes' Studien der Optik an, zusammen studierten die beiden Vesalius' Fabrica, ja Hobbes soll sogar anatomischen Zergliederungen beigewohnt haben, die Petty durchführte (vgl. Zimmer 2004, S. 99). Später wurde Petty Professor der Anatomie in Oxford und Gründungsmitglied der Royal Society (vgl. McCormick 2006). Als Arzt der britischen Armee unter Cromwell traf Petty 1652 zum ersten Mal in Irland ein. Er hatte hier den Auftrag, die Pest zu bekämpfen und eine kartografische Studie auszuführen, welche der Aneignung des Landes durch die englischen Besetzer diente; eroberte irische Territorien wurden damals englischen Anspruchsstellern zugeteilt (vgl. Jordan 2007, S. 5, 45). Auch Petty selbst eignete sich in dieser Zeit große Grundstücke an (vgl. Zimmer 2004, S. 111). Fast vierzig Jahre später, 1691, vier Jahre nach Pettys Tod,

8 Gott liess dem zweifelnden Hiob das Seeungeheuer „Leviathan“ in einem Unwetter erscheinen, um die eigene Macht zu offenbaren. 
wurde dessen Studie Political anatomy of Ireland publiziert. Petty, der sich für den volkswirtschaftlichen Schaden von Krankheiten interessierte (Rosen 1958, S. 115), vergleicht in seiner Studie unter anderem die Stärke des Pestausbruches in Irland und London um 1650 (vgl. Rosen 1958, S. 115). In der Einleitung schreibt Petty, er habe im ersten Essay zur Politischen Anatomie in Anlehnung an Francis Bacon - dessen Assistent wiederum Hobbes gewesen war - zwischen dem „Body Natural“ und dem „Body Politick“ eine scharfsinnige Parallele gezogen (vgl. Petty 1899, S. 129). ${ }^{9}$ Als Versuchskaninchen habe ihm dabei das junge Irland gedient, denn hier seien die Machenschaften des Staates nicht sehr kompliziert; zudem, so führt Petty seine kolonial inspirierte Körpermetaphorik fort, sei er mit Irland vertraut, seit dieses ein Embryo gewesen sei. Petty eröffnete einen statistischen Zugang zu ökonomischen Fragen, wobei er zugleich eine politische Perspektive auf die Bevölkerung entwarf. Seiner Meinung nach hätte der ärmliche und kränkliche Zustand Irlands durch eine günstigere Proportion zwischen englischer und irischer Bevölkerung verbessert werden können, weshalb er zur Verheiratung englischer Frauen mit irischen Männern riet. Um die irische Arbeitskraft zu vergrössern, schlug Petty die Reduzierung von Feiertagen, Klöstern und Bierstuben vor (vgl. Petty 1899, S. 223). Vor allem aber sollten Emigrationswillige einen Vorteil darin erblicken, nach Irland zu ziehen, um so dem Mangel an Menschen abzuhelfen, dem nach Petty grössten und fundamentalsten Mangel dieses Landes (vgl. Petty 1899, S. 223). Andererseits forderte er, die Iren seien nach England zu transplantieren (vgl. Petty 1986, S. 559, 568). David Armitage meint dazu: „This would have left some 300.000 people in Ireland to administer the country as a cattle-ranching dependency of England“ (Armitage 2000, S. 151). Mit seiner Vision der Säuberung Irlands von den Iren als notwendige Vorbedingung einer Produktivitätssteigerung kann er als „,Vordenker“ für Selektion gesehen werden. Er begreift die Bevölkerung als Ressource, allerdings vertrat er nicht einen biologischen Determinismus (der sich historisch gesehen auch erst später durchsetzte), können doch beispielsweise irische Männer durch die Verheiratung mit englischen Frauen umerzogen werden. Andererseits ist die biologische Grundierung seiner Sprache im wörtlichen Sinne frappierend.

\section{Biopolitik}

Foucault, der die politische Theorie von ihrer Fixierung auf die Souveränität befreien wollte (Foucault 2001, S.200), gilt als einer der Präger des Begriffes Biopolitik. Nach Michel Foucault wurde die Vorstellung, dass die Kraft eines Staates von der Anzahl seiner Bewohner abhängt, früh im Mittelalter formuliert. Sie habe indes erst im 17. Jahrhundert einen genauen Sinn angenommen, in dem Moment, als man sich das Problem stellte, wie viele Menschen ein bestimmtes Territorium tatsächlich brauche (vgl. Foucault 2004a, S. 465). Die Geburt der Biopolitik im Sinne einer Regulierung und Verbesserung der Bevölkerung verortet er im 18. Jahrhundert (vgl. Foucault 2004a, S. 27, 145, 147, 159, 164). Die biopolitisch optimierte Bevölkerung wiederum ist nach Foucault u. a. ein Ziel gouvernementaler Führung (vgl. Opitz 2004, S. 38), was er als eine Art des Regierens

9 Vergleiche zwischen dem Körper und dem Staat sind bekanntlich nicht Pettys Erfindung, sondern gehen u. a. bereits auf Platon zurück. 
begreift, welche auf das Einverständnis der Regierten setzt. Den Begriff der Gouvernementalität führte Foucault in seiner gegenwärtigen Verwendung Ende der 1970er Jahre ein. Er grenzte dabei Souveränität, Disziplinarmacht und Gouvernementalität analytisch von einander ab. Die jeweils voneinander differenzierbaren Modelle sind nicht als chronologisch folgend, sondern als Akzentverschiebung zu denken. Die These Foucaults lautet, die letzte Staatstheorie fände sich bei Hobbes, denn „Locke entwickelt nicht mehr eine Theorie des Staates, sondern der Regierung" (2004b, S. 133). Auf Hobbes folge Locke, und bei diesem werde die Begründungsbedürftigkeit des Staates von den Naturgesetzen der Ökonomie abgelöst (vgl. Foucault 2004b, S. 133). Foucault bringt den Begriff der Gouvernementalität in Verbindung mit Locke, genauer gesagt mit dessen Schrift Two treatises of government. Allerdings kann das Frontispiz des Leviathan - ausgehend von unseren Überlegungen zu Hobbes' Assistenten Petty, zur Schnabelmaske und der Seuchenpolitik -als Einsatzstelle gesehen werden, wo ein sanitarischer Schutz des Staatskörpers und damit ein Blick auf die Bevölkerung ins „Bild des Politischen“ rückt. In Bezug auf den Leviathan sollte indes nicht übersehen werden, dass hier die Metaphorik des Körpers einerseits gebraucht, andererseits aber in bestimmter Hinsicht auch gebrochen wird. Hobbes denkt den Staat als natürlich aber zugleich als künstlich, beschreibt er ihn doch auch als Maschine (vgl. Skinner 1996, S. 387): Er versteht Natur und Kultur nicht als Gegensätze (vgl. Stollberg-Rilinger 1986, S. 49).

Foucault stellte dem Modell des Ausschlusses, wie es auf Leprakranke angewendet wurde, jenes des Einschlusses gegenüber, das er in der Pestpolitik verwirklicht sah: Die Kranken würden nun nicht mehr verbannt, vielmehr weise man ihnen einen festen Platz zu (vgl. Foucault 1999, S. 42). Dabei werde nicht mehr eine dichotomisch operierende Grenze zwischen Kranken und Gesunden gezogen, sondern es werde ein System von feinen Differenzen etabliert. Dies führe zu einer ständigen Kontrolle des einzelnen Individuums, so dass beispielsweise die noch gesunden Familienmitglieder unter ständige Beobachtung gestellt würden. Mit diesem Modell, das sich an der Pestpolitik orientiere, sei es gleichzeitig zur Erfindung der „technologies positives du pouvoir“ gekommen (vgl. Foucault 1999, S. 44). Später wiederum wurde diese Pestpolitik nach Foucault von einem „Pocken-Modell der Macht“ abgelöst. Dieses basiere, so Philipp Sarasin, im Wesentlichen darauf, „dass die Macht den Traum aufgibt, die Pathogene, die Eindringlinge, die Krankheitskeime vollständig auszumerzen“" (2005/2006, S. 162). Damit wird auch die Vorstellung von der Notwendigkeit hermetischer Grenzen relativiert; das Prinzip der Impfung funktioniert durch Integration, nicht durch Isolierung (vgl. Foucault 2004a, S. 93). Diese soll Zirkulation ermöglichen, eine solche Sanitätspolitik dient hier also gerade einer territorialen Offenhaltung. Die Logik dieses Sicherheitsmechanismus' bringt Alison Bashford treffend auf den Punkt: „That is, far from working on a preventive model of separation and the maintenance of lines of hygiene, vaccination was precisely about contact, mixing and dissolving the foreign ,other" within the self"'(2001, S. 40). Gouvernementalität und Biopolitik können dabei durchaus in einem Spannungsverhältnis zueinander stehen: Eine Form liberaler Staatlichkeit, die sich mit der Freiheit der Individuen Grenzen setzt, steht dabei einer totale Verwertung der Biomasse entgegen (vgl. Sarasin 2005).

Auf dem Emblem des politischen Körpers werden in einem Spannungsverhältnis Souveränität, Disziplinarmacht und eine beginnende Biopolitik sichtbar. Die Machtformation der Souveränität zeichnet sich nach Foucault durch ein Ausüben von Zwang aus, sie funk- 
tioniert als Zugriffsrecht. Die Disziplinarmacht wirkt auf die einzelnen Körper und diszipliniert diese; dies ist auf dem Frontispiz sehr deutlich ersichtlich, der Soldat steht hier als Sinnbild für den disziplinierten Körper. Biopolitik ist nach Foucault eine Politik, die auf das Leben zielt, und zwar der Gesamtbevölkerung; dieses Leben gelte es zu steigern, zu verbessern. Es ist nun die Machtformation der Gouvernementalität, die nach Foucault mit dem Modus der Biopolitik operiert, sich jedoch nicht auf Biopolitik beschränken lässt. In gouvernementalen Machtstrukturen verbindet sich das Regieren anderer mit der Selbstregierung. Foucault bringt dies im Speziellen mit einer liberalen Form des Regierens in Verbindung, die auf das Einverständnis der Regierten setzt und auf deren Freiheit: Gerade diese wird auch ökonomisch produktiv gemacht (vgl. Foucault 2004a, S. 162). Für Hobbes ist hingegen nicht Freiheit, sondern Sicherheit das Ziel des Staates, auch wenn nach Hobbes rationale Individuen dem Gesellschaftsvertrag freiwillig zustimmen.

Foucault sieht in „Les anormaux“ (vgl. Foucault 1999, S.41-44) die Pestpolitik als Beginn einer Machtformation, die nicht nur als Unterdrückungsinstanz, sondern produktiv wirkt, lebensverlängernd, auch wenn er da noch nicht auf den Begriff der Gouvernementalität zurückgreift und seine Analyse dabei noch stärker vom Individuum ausgeht und weniger von der Gesamtbevölkerung. Später bringt Foucault gouvernementale Sicherheitsdispositive mit den Beispielen der Impfung und der Statistik vor allem mit der Bekämpfung der Pocken in Verbindung. ${ }^{10}$ Meine Ausführungen zu Hobbes' Assistenten Petty zeigen indes, dass bereits dieser u.a. durch das Fördern einer bestimmten Heiratspolitik die Bevölkerung in ihrer Qualität zu verbessern trachtete; Petty betrachtete die Bevölkerung als etwas Gemachtes und nicht einfach Gegebenes. Was mir besonders bedeutsam erscheint, ist, dass ausgehend von einer solchen Betrachtungsweise auf dem Emblem des politischen Körpers eine beginnende Biopolitik 1651 wortwörtlich ins „Bild“ des Politischen rückt, ${ }^{11}$ während der Beginn des biopolitischen Zeitalters in Foucault-Rezeptionen bisher oft erst auf ein- bis zweihundert Jahre später datiert wird. Die Schnabelmasken auf dem Frontispiz des Leviathan können damit als Aufhänger für biopolitisch akzentuierte Lektüren dienen.

\section{Politische Optik}

Hobbes - der ja Herrschaft gerade mit dem Verweis auf den Schutz des Lebens legitimierte (vgl. Bürgin 2008, S.171) - wurde übrigens nach seiner Rückkehr aus dem französischen Exil mit dem Londoner Pestausbruch von 1666 in Verbindung gebracht. Als Folge der damals geäußerten Atheismusvorwürfe wurden Neudrucke des Leviathan verboten. Selbst wenn aber Hobbes bereits früher mit der Pest konfrontiert worden war,

10 Es besteht vielleicht auch ein gewisses Spannungsverhältnis zwischen den idealtypischen Denkmodellen, die Foucault generiert - dass eben die Bekämpfung der Lepra, der Pest und der Pocken paradigmatisch verstanden werden kann - und der historischen Konkretisierung seiner Arbeit.

11 Mir ist klar, dass das Argument mit Agambens Begriff der Biopolitik einfacher wäre, sieht er doch eine (allerdings von anderen auch kritisierte) notwendige Verbindung zwischen der Souveränität und Biopolitik. Dazu auch Vaughan-Williams 2009. 
bleibt die Frage offen, ob das Detail der Schnabelmaske auf dem Frontispiz Hobbes' eigener Vorschlag war.

In seiner „Critique du De mundo de Thomas White“ schreibt Hobbes, die Zergliederung eines Objektes verlängere den Genuss, wohingegen die immer gleiche Sicht oder die Wiederholung des gleichen musikalischen Tons bald die Ohren und Augen beleidige (vgl. Hobbes 1976, S. 464; Daston und Park 1998, S. 314). Ein Objekt, welches zu klein sei, um bewusst gesehen zu werden, wirke dennoch auf die Sehorgane: Selbst wenn ein einzelnes Sandkorn aus Distanz nicht wahrnehmbar sei, würde der Sandhaufen als Ganzes dennoch sichtbar. Hobbes erklärt diesen Umstand damit, dass von jedem Einzelteil eine Aktivität, eine Wirkung ausgehe (vgl. Hobbes 1839, S.342). Hobbes war also von der Wirkmächtigkeit kaum wahrnehmbarer Details überzeugt. Die Frage, ob und inwieweit er selbst Einfluss genommen hat auf die Gestaltung des Titelblattes, ist zwar nicht definitiv zu klären, doch es lassen sich Argumente aufführen, die eine solche Annahme plausibel erscheinen lassen. Hobbes betrieb selbst optische Studien (vgl. Prins 1996). In Zusammenhang mit seiner ,politischen Optik“ ist vor allem die in einem Briefwechsel von 1650 mit dem Schriftsteller William Davenant genannte Beschreibung eines Experiments wichtig, in welchem verschiedene menschliche Figuren durch Speziallinsen zu einer ganzen Person zusammengefügt wurden (vgl. Malcolm 2002; Bredekamp 2003, S. 89; Windisch 1997). „I believe, Sir, you have seen a curious kind of perspective, where he that looks through a short hollow pipe upon a picture containing diverse figures sees none of those that are there painted, but some one person made up of their parts, conveyed to the eye by the artificial cutting of a glass" (1840, S.457). Bemerkenswert ist, dass Hobbes im Leviathan auf diese optischen Instrumente zu sprechen kommt, indem er schreibt, dass Menschen von Natur aus in ihrer Leidenschaft und Selbstliebe über „multiplying glasses“ verfügten, wodurch jede Steuer als grosse Belastung erscheine, ihnen jedoch ,prospective glasses“ fehlten - nämlich die Wissenschaft von der Moral und vom Staat - „um von ferne die elenden Zustände zu sehen, die über ihnen hängen und ohne diese Abgaben nicht abgewendet werden können" Hobbes (1966, S. 144). Dieses Kompositionsbild, das Hobbes bei seiner Arbeit am Leviathan wahrscheinlich inspirierte, existierte jedoch nicht als ein Bild mit einem physisch konkreten Körper, sondern vielmehr als ein visuelles Konstrukt, als Resultat eines optischen Experiments (vgl. Malcolm 2002, S. 222): „like the optical scientist who shows that illusions are created by the operation of natural laws, not demons, Hobbes shows that the sovereign power exists not as a supernatural entity or sacral authority but as a consequence of the rational acts of will of the people" (Malcolm 2002, S. 227). Die Analogie von Staat und optischem Experiment darf indes nicht dahingehend verstanden werden, dass Souveränität das Resultat einer Sinnestäuschung sei, vielmehr kann damit die Tatsache betont werden, dass der Staat gemacht, nicht gegeben ist. Allerdings folgt die politische Macht nicht nur dem Gebot der Sichtbarkeit, sondern auch dem Prinzip der Arcana imperii. Die Formel bezeichnet die geheimen, verborgenen und unsichtbaren Mittel der Macht, deren Verschleierung mit Vorhang auf dem Frontispiz angedeutet ist. Nach Horst Bredekamp ist dieser nach dem Vorbild jenes Schleiers gestaltet, welcher das Tabernakel des Alten Testaments verborgen gehalten habe (Bredekamp 2007, S.32). 


\section{Bild und Gewalt}

„Ohne das Bild des ,Leviathan“ bleibt Hobbes politische Theorie so missverständlich wie die Dunkelheit jener Sprache, die er als Verfall der semantischen Verbindlichkeit kritisiert“", schreibt Bredekamp (Bredekamp 2003, S. 131). Seine bekannte Argumentation folgt der Hobbesschen Rhetorik, die vor der Macht der Metaphern, der Rede und der Schrift warnt. Zugleich jedoch insistiert Bredekamp in seiner Interpretation von Hobbes auf der politischen Notwendigkeit von Bildern. Ohne Schwert seien Verträge blosse Worte; es brauche aber nebst den Waffen auch ein Bild, um die Rhetorik des Gesellschaftsvertrags zu bändigen. Tatsächlich spricht Hobbes von der Notwendigkeit einer sichtbaren Gewalt, um die Menschen im Zaum zu halten (vgl. Hobbes 1966, S. 131). Eine Sichtbarkeit der Macht bedeutet aber nicht zwangsläufig, dass diese über Bilder erzeugt wird. Auch macht Bredekamp die Notwendigkeit einer Sichtbarkeit der Macht im Leviathan prominenter, als sie von Hobbes verhandelt wird. Denn meiner Meinung nach liegt die Betonung in Hobbes' Formulierung ,wenn es keine sichtbare Gewalt gibt, die sie im Zaume zu halten und durch Furcht vor Strafe an die Erfüllung ihrer Verträge und an die Beachtung der natürlichen Gesetze zu binden vermag“ (1966, S. 131) eher auf „Gewalt“ denn auf „sichtbar“: Hobbes spricht im Leviathan nur sehr selten von einer notwendigen Sichtbarkeit, während er das Erfordernis eines physischen Gewaltmonopols sehr stark betont. Wenn auch die Angst vor dem Einsatz der Gewalt nach Hobbes eine Notwendigkeit ist, so wird diese Angst laut Leviathan nicht primär in visueller Weise erzeugt. Während also Hobbes vor allem verdeutlicht, dass Worte für Verträge nicht genügen, dass vor allem Waffen nötig seien, um Einhaltung der Verträge zu erzwingen, akzentuiert Bredekamp in seiner Lektüre von Hobbes die Wichtigkeit des Bildes. Mir scheint, dass Bredekamp Hobbes hier vielleicht zu sehr durch die Linsen von Louis Marin liest (vgl. Marin 2005). ${ }^{12}$ Es könnte allerdings argumentiert werden, die Annahme einer radikalen Abhängigkeit des Souveräns von seiner Repräsentation strukturiere implizit die Hobbessche Argumentation.

\section{Hobbes' Bildtheorie und die Grenzen des souveränen Staates}

Hobbes entwarf im Leviathan selbst eine „Bildtheorie“. Entweder sei ein Bild ein Abbild eines sichtbaren Gegenstands - wie beispielsweise bei einer Spiegelung im Wasser; hier bestehe ein Ähnlichkeitsverhältnis - oder ein Bild vertrete ein Ding; da gelte hingegen keine Ähnlichkeitsbeziehung. ${ }^{13}$ Hobbes schreibt: „So kann ein irdischer Souverän als das Bild Gottes bezeichnet werden“ Hobbes (1966, S. 496). Dies wiederum bedeute nicht, dass man sich von Gott ein ,mimetisches“ Bild machen und dieses als authentische Darstellung ansehen dürfe. Hobbes kritisiert diese Art von religiöser Bildverehrung an meh-

12 Louis Marin hat gezeigt, wie eine permanente Präsenz des Monarchen auch bei physischer Absenz durch zirkulierende Bilder produziert werden sollte. Der Repräsentationseffort des Sonnenkönigs beispielsweise wäre somit auch als Antwort auf jene Krise zu verstehen, die durch die Kollision von absoluten Staatsvorstellungen und aufklärerischen Tendenzen provoziert wurde: Bildpotenz sollte sozusagen reale Impotenz verdecken.

13 Meistens allerdings fungieren Bilder laut Hobbes sowohl als Abbild wie auch als Vertretung. 
reren Stellen (vgl. Hobbes 1966, S.279, 497, 498, 501, 502, 505) mit dem bekannten Argument, dass jede Vorstellung begrenzt, Gott aber das Unbegrenzte sei (vgl. Hobbes 1966, S. 495). Hobbes geht weiter auf den Unterschied zwischen Sichtbarkeit und Bildlichkeit kurz ein, als er ausführt, „Gott dienen aber heisse, seine Vorstellung von ihm anderen sichtbar zu machen“, beispielsweise im Gottesdienst (Hobbes 1970, S. 299). ${ }^{14}$

Das Bild des Leviathan könnte nach Hobbes’ Logik ebenfalls weniger als Abbild, denn als Stellvertreter verstanden werden. Selbst wenn der bildlich dargestellte Souverän allerdings als sehr mächtig und auf dem Frontispiz von 1651 als oben ungerahmter erscheint, zeichnet sich der von Hobbes begründete souveräne Einzelstaat durch (gewisse) Grenzen aus, die allerdings selten explizit verhandelt werden. Implizit erschliesst sich jedoch eine klare Trennung zwischen den souveränen Einzelstaaten, (die Autorität des Staates wird dabei als territorial begrenzt gedacht, geht doch Hobbes davon aus, dass sich die Staaten untereinander im Naturzustand befinden), zwischen innen und aussen, privatem Glauben und öffentlichen Äußerungen. Auch das Frontispiz des Leviathan operiert durchgehend mit Oppositionspaaren und evidenten Grenzen: Der Bildraum ist klar aufgeteilt zwischen oben und unten, links und rechts, staatlicher und kirchlicher Macht, wobei sich die Religion nicht über staatlich gesetzte Grenzen hinwegsetzen darf. Gleichzeitig ist Hobbes ein Denker, der Grenzen zuweilen abzubauen vermag, so beispielsweise in Bezug auf innergesellschaftliche Grenzziehungen.

\section{Soldaten, Seuchenärzte und Sicherheitsversprechen}

Es sind nun nicht nur Seuchenärzte, sondern auch Soldaten in der menschenleeren Stadt zurückgeblieben. Soldaten und Seuchenärzte üben eine verwandte Funktion aus: Beide sollen den Staatskörper schützen. Der Gesellschaftsvertrag sorgt zwar innerhalb eines Staates für Frieden, dennoch ist der Krieg nicht aufgehoben, sondern nur an die Grenzen gedrängt. Zwischen den Staaten herrscht nach Hobbes der Naturzustand. Diese verhalten sich wie Gladiatoren: ,sie richten ihre Waffen gegeneinander und lassen sich nicht aus dem Auge - das heißt, sie haben ihre Festungen, Garnisonen und Geschütze an den Grenzen ihrer Reiche und ihre ständigen Spione bei ihren Nachbarn" Hobbes (1966, S. 97, 98). Im Leviathan ächtet Hobbes zwar den Bürgerkrieg, hingegen bewertet er die Anarchie im zwischenstaatlichen Bereich nicht als negativ, denn es könne dadurch der Fleiss der Staatsangehörigen aufrecht erhalten werden, ja sogar noch gefördert werden, weshalb daraus nicht das Elend resultiere, ,das die Freiheit von Einzelmenschen begleitet" Hobbes (1966, S.98). Auseinandersetzungen im internationalen Bereich sind also immer noch möglich, ja sogar wahrscheinlich, und dieser Umstand bedroht das Leben

14 Ich habe mich hier auf die Übersetzung der jüngeren lateinischen Fassung gestützt. Hingegen wird in der englischen Leviathan Ausgabe noch deutlicher, dass ein Sichtbarmachen nicht zwangsläufig visuell zustande kommen muss. Hier heißt es auf Seite 238: „the external signs appearing in the words, and actions of men, are called worship“ (Hobbes 1996). Die Hobbessche Definition von signs, auf die Bredekamp rekurriert, um eine Bildakttheorie zu formulieren, bezieht sich bei Hobbes demnach auf vieles, beispielsweise auch Worte und Handlungen und nicht spezifisch auf Bilder. 
des Individuums weiterhin. Der Staat kann sein Sicherheitsversprechen nicht vollständig einlösen (vgl. Cheneval 2002). „Jedermann ist von Natur aus verpflichtet, soweit es in seiner Macht steht, im Krieg die Autorität zu schützen, durch die er in Friedenszeiten geschützt wird“" Hobbes (1966, S. 536). Die Verpflichtung der Staatesangehörigen gegenüber dem Souverän dauert nach Hobbes allerdings nur solange, wie dieser sie aufgrund seiner Macht schützen kann, und nicht länger (vgl. Hobbes 1966, S. 171, 254, 537). Für den angeworbenen Soldaten sieht die Situation etwas anders aus. Dieser ist zwar zur Verteidigung des Souveräns verpflichtet (vgl. Hobbes 1966, S. 169), jedoch schreibt Hobbes an anderer Stelle, selbst dieser sei nicht zum Opfertod gezwungen (vgl. Hobbes 1966, S. 159). Die Figur des Leviathan ist demnach nicht ohne innere Spannung; kann der individuelle Körper, das Leben des Einzelnen, nicht geschützt werden, zerfällt der Souverän. Hier zeigt sich allerdings auch eine interessante Differenz zwischen der Schrift des Leviathan und seinem Bild: Während in der Schrift des Leviathan die potentielle Auflösung des Leviathan angelegt ist, wird im Titelbild die potentielle Auflösung des Individuums im Staatskörper betont, verschwinden die Menschen doch zum Teil in der Schraffur, so beispielsweise im Unterarm. In gewisser Weise erscheint so die Schrift liberaler, stärker auf die Individualrechte abstützend als das Bild des Leviathan. Vielleicht hat sich auch Michel Foucault stärker vom Bild als von der Schrift des Leviathan beeindrucken lassen?

\section{Fazit}

Ich kann hier naturgemäß nicht alles ausführen, was zum Frontispiz oder der Schrift des Leviathan gesagt werden könnte; so konnte ich beispielsweise nicht auf die Entstehungsbedingungen des Titelbildes eingehen und auch nicht auf seine Vor- und Nachbilder; Horst Bredekamp hat dies zudem in seinen paradigmatischen Studien aufgezeigt. Auf Bilder lassen sich bekanntlich ganz verschiedene Blicke werfen; am spannendsten schien mir hier ein Wechselspiel zwischen großer Nähe und weiter Distanz. Ob ein Detail als trivial oder aufschlussreich gesehen wird, ist kontextabhängig. Dieser Kontext ist jedoch nicht gegeben, kann nicht vorausgesetzt werden, sondern muss geschaffen werden. In der hier vorgeschlagenen Sichtweise wurden die Schnabelmasken zum Schwer- und Drehpunkt der Interpretation. Als Wahrnehmungsfilter verändern sie die Sicht auf den dargestellten Staatskörper. Sie lenken den Blick auf die Gewaltsamkeit der Grenzziehung, die nötig war, um die souveräne Ganzheit und Abgeschlossenheit des Staatskörpers herzustellen. Die bildlich suggerierte inkorporierende Inklusion auf dem Frontispiz des Leviathan lässt diese Exklusionen auf den ersten Blick nicht erahnen. Der von den Schnabelmasken gelenkte Blick macht weiter den programmatischen Zusammenhang von Souveränität und Sanität sichtbar: Städtische bzw. staatliche Strukturen begannen sich insbesondere im Krieg und im seuchenpolitischen Ausnahmezustand (vgl. Slack 1985) herauszubilden. Medizinische Dispositive waren unter anderen Faktoren (militärischen, ökonomischen etc.) wichtig für die Etablierung und Legitimierung von Grenzen. Allerdings sollen hier auch die Widersprüche und Spannungsverhältnisse nicht unsichtbar gemacht werden, die gerade auch in Bezug auf Grenzziehungen zwischen Souveränität, Disziplinar-und Biomacht bestehen: „Whereas disciplinary power isolates, concentrates and is essentially protectionist, by contrast biopower is said to be centrifugally oriented in favour of expansion, 
circulation and movement" (Vaughan-Williams 2009, S. 80). Für Foucault sind Biopolitik und der Liberalismus, den er als Rationalisierung der Regierungsausübung versteht (Foucault 2004b, S. 436), intrinsisch miteinander verbunden. Foucault stellt Hobbes zudem im Gegensatz zu Locke nicht in die Tradition des Liberalismus; doch wird Hobbes in anderen Lesarten auch liberaler ausgelegt und als Anfangspunkt einer sukzessiven Entfaltung von Rechtsansprüchen gegenüber dem Staat verstanden (Münkler 2003).

Der biopolitische Kontext, der aus der hier vorgeschlagenen Perspektive insbesondere über Hobbes' Assistenten William Petty ins Bild des Politischen rückt, weist dabei mit von Anfang an einen kolonialen Referenzrahmen auf, was übrigens auf dem Frontispiz von De Cive noch viel deutlicher wird, was hier allerdings nicht weiter ausgeführt werden konnte (Falk 2011b). Der Pestarzt findet dabei sein Pendant im Soldaten: Beide riskieren in ihrem Einsatz ihr Leben, beide verweisen aber zugleich auf die Fragilität des Staates, sie markieren die Grenzen des Leviathan: Kann der Schutz des Lebens nicht gewährleistet werden, sei es durch Kriege, ausgelöst durch den Naturzustand des bellum omnium contra omnes zwischen den Staaten, sei es durch den seuchenpolitischen Ausnahmezustand, zerfällt der Staat. Die Schnabelmasken sind für das Bild des Leviathan bzw. des Staatskörpers wichtig, weil sie sich hier zeigen (nicht aber in Hobbes' Text). ${ }^{15}$

Die hier vorgeschlagene Sichtweise wird indes erst durch den Kurzschluss verschiedener Zeitlichkeiten möglich, in einem Augenblick der Geschichte also, in dem mit Benjamin gesprochen ,das Gewesene mit dem Jetzt blitzhaft zu einer Konstellation zusammentritt“ (1983, S. 576-577). Erst in der Jetztzeit, vor dem Hintergrund zeitgenössischer Diskurse, sind die Schnabelmasken auf dem Frontispiz offenbar zur Lesbarkeit gelangt. ${ }^{16}$

\section{Literatur}

Agamben, Giorgio. 2004. Ausnahmezustand. Frankfurt a. M.: Suhrkamp.

Armitage, David. 2000. The ideological origins of the British Empire. Cambridge: Cambridge University Press.

Bashford, Alison. 2001. Foreign bodies: vaccination, contagion and colonialism in the nineteenth century. In Contagion: historical and cultural studies, Hrsg. Alison Bashford, und Claire Hooker, 39-60. London: Routledge.

Bazin, Janine. 1971. L'évolution du costume de médicine de peste en Europe de 1348-1720. Paris: Ed. Médicales et Univ.

Benjamin, Walter, und Rolf Tiedemann. 1983. Das Passagen-Werk. Frankfurt a. M.: Suhrkamp.

Boeckl, Christine M. 2000. Images of plague and pestilence. Iconography and iconology. Kirksville: Truman State UP.

Bohn, Cornelia. 2006. Passregime: Vom Geleitbrief zur Identifikation der Person. AWR-Bulletin. Viertelsjahrschrift für Flüchtlingsfragen 53 (4): 254-269.

Brandt, Reinhard. 2000. Thomas Hobbes: Das Titelblatt des Leviathan. In Philosophie in Bildern, Hrsg. Reinhard Brandt, 312-330. Köln: DuMont Buchverlag.

15 Die Schnabelmasken lassen sich dabei als etwas beschreiben, dessen Relevanz unabhängig von Hobbes' eigener Intention gegeben ist.

16 Wobei Lesbarkeit hier metaphorisch verstanden werden soll und sich gerade nicht auf die Lektüre eines Textes beschränkt. 
Bredekamp, Horst. 2003. Thomas Hobbes „Der Leviathan“. Das Urbild des modernen Staates und seine Gegenbilder 1651-2001. 2., stark verändert. Aufl. Berlin: Akademie.

Bredekamp, Horst. 2007. Thomas Hobbes's visual strategies. In The Cambridge companion to Hobbes's Leviathan, Hrsg. Patricia Springborg, 29-60. Cambridge: Cambridge University Press.

Bredekamp, Horst. 2009. Behemoth als Partner und Feind des Leviathan. Zur politischen Ikonologie eines Monstrums. Leviathan. Berliner Zeitschrift für Sozialwissenschaft 37 (3): 429-475.

Broquet, C. 1911. Le masque dans la peste. Présentation d'un modèle de masque antipesteux. Bulletin de la société de pathologie exotique. Institut Pasteur. 636-645.

Brossollet, Jacqueline. 1979. Costume del medico della peste nei secoli XV et XVI. In Venezia e la Peste 1348/1797, Hrsg. Jacqueline Brossollet, 63-65. Venezia: Marsilio Ed.

Brusatin, Manlio. 1981. Il muro della peste: Spazio della pietà e governo del lazzaretto. Venezia: Cluva.

Bürgin, Ariane. 2008. Endliches Subjekt: Gleichheit und der Ort der Differenz bei Hobbes und Rousseau. München: Wilhelm Fink.

Cheneval, Francis. 2002. Philosophie in weltbürgerlicher Bedeutung. Über die Entstehung und die philosophischen Grundlagen des supranationalen und kosmopolitischen Denkens der Moderne. Basel: Schwabe.

Daston, Lorraine J., und Katharine Park. 1998. Wonders and the order of nature 1150-1750. New York: Zone Books.

Dinges, Martin. 1995. Pest und Staat: Von der Institutionengeschichte zur sozialen Konstruktion? In Neue Wege in der Seuchengeschichte, Hrsg. Thomas Schlich, und Martin Dinges, 71-104. Stuttgart: Franz Steiner.

Falk, Francesca. 2011a. Eine gestische Geschichte der Grenze. Wie der Liberalismus an der Grenze an seine Grenzen kommt. Paderborn: Wilhelm Fink.

Falk, Francesca. 2011b. Postkoloniale Perspektiven auf die politische Philosophie. Thomas Hobbes' horror vacui und John Lockes leeres Land. In Tierische (Ge)Fährten. Historische Anthropologie: $\mathrm{xx}$.

Fazzini, Gerolamo. 2004. Venezia - Isola del Lazzaretto Nuovo. Venezia: Archeoclub d'Italia.

Foucault, Michel. 1999. Les anormaux. Cours au Collège de France (1974-1975). Paris: Gallimard.

Foucault, Michel. 2001. Gespräch mit Michel Foucault. In Schriften in vier Bänden. Dits et Ecrits, Hrsg. Daniel Defert, und François Ewald, 186-212. Frankfurt a. M.: Suhrkamp.

Foucault, Michel. 2004a. Geschichte der Gouvernementalität I. Sicherheit, Territorium, Bevölkerung. Vorlesung am Collège de France 1977-1978. Frankfurt a. M.: Suhrkamp.

Foucault, Michel. 2004b. Geschichte der Gouvernementalität II. Die Geburt der Biopolitik. Vorlesung am Collège de France 1978-1979. Frankfurt a. M.: Suhrkamp.

Ginzburg, Carlo. 2008. Welt der Leviathane. Furcht, Verehrung, Schrecken - Thomas Hobbes' Politische Theologie. Lettre International 83:23-27.

Groebner, Valentin. 2002. Der Schein der Person. In Quel corps? Eine Frage der Repräsentation, Hrsg. Hans Belting, Dietmar Kamper, und Martin Schulz, 307-323. München: Wilhelm Fink.

Hobbes, Thomas. 1839. The english works of Thomas Hobbes of Malmesbury. London: John Bohm.

Hobbes, Thomas. 1840. The english works of Thomas Hobbes of Malmesbury. London: John Bohm.

Hobbes, Thomas. [1651]1966. Leviathan oder, Stoff, Form und Gewalt eines kirchlichen und bürgerlichen Staates. Frankfurt a. M.: Suhrkamp.

Hobbes, Thomas. [1670]1970. Leviathan. Erster und zweiter Teil. Stuttgart: Philipp Reclam Junior.

Hobbes, Thomas. [1642]1976. Thomas White's De mundo examined. London: Bradford University Press. 
Hobbes, Thomas. [1651]1996. Leviathan, or the matter, form, and power of a commonwealth ecclesiastical and civil. Oxford: Oxford University Press.

Jordan, Thomas Edward. 2007. Sir William Petty, 1623-1687. The genius entrepreneur of seventeenth-century Ireland. Lewiston: Edwin Mellen Press.

Kantorowicz, Ernst Hartwig. 1992. Die zwei Körper des Königs. Eine Studie zur politischen Theologie des Mittelalters. Stuttgart: Klett-Cotta.

König, Helmut. 2008. Politik und Gedächtnis. Weilerswist: Velbrück Wissenschaft.

Landwehr, Achim. 2007. Die Erschaffung Venedigs. Raum, Bevölkerung, Mythos, 1570-1750. Paderborn: Ferdinand Schöningh.

Leven, Karl-Heinz. 2001. Mensch - Umwelt - Seuchen: Wechselwirkungen zwischen Krankheit und Lebensumwelt seit der Antike. In Ökohistorische Reflexionen. Mensch und Umwelt zwischen Steinzeit und Silicon Valley, Hrsg. Kurt W. Alt, Natascha Rauschenberger, 75-98. Freiburg im Breisgau: Rombach.

Levine, David A., und Mai Ekkehard. 1991. I Bamboccianti. Niederländische Malerrebellen im Rom des Barock. Milano: Electa.

Malcolm, Noel. 2002. The title page of Leviathan, seen in a curious perspective. In Aspects of Hobbes, Hrsg. Noel Malcolm, 200-229. Oxford: Oxford University Press.

Manow, Philip. 2007. Sexualität und Souveränität - Neue Nachrichten vom Vor- und Nachleben des Leviathan-Frontispizes. Leviathan. Berliner Zeitschrift für Sozialwissenschaft 35 (4): 470-494.

Marin, Louis. 2005. Das Porträt des Königs. Berlin: Diaphanes.

Mccormick, Ted. 2006. Alchemy in the political arithmetic of Sir William Petty (1623-1687). Studies in History and Philosophy of Science 37 (2): 290-307.

Münkler, Herfried. 2003. Politische Ideengeschichte. In Politikwissenschaft. Ein Grundkurs, 103131. Reinbek bei Hamburg: Rowohlt.

Noack, Thorsten. 2009. Der Narr wird verrückt. Medizinische Konzepte des Wahnsinns im Europa der Frühen Neuzeit. In Narren - Masken - Karneval. Meisterwerke von Dürer bis Kubin aus der Düsseldorfer Graphiksammlung ,, Mensch und Tod“, Hrsg. Stefanie Knöll, 10-17. Regensburg: Schnell \& Steiner.

Opitz, Sven. 2004. Gouvernementalität im Postfordismus. Macht, Wissen und Techniken des Selbst im Feld unternehmerischer Rationalität. Hamburg: Argument.

Orwin, Clifford. 1988. Stasis and plague: Thucydides on the dissolution of society. Journal of Politics 50 (4): 831-847.

Palmer, Richard J. 1978. The control of plague in Venice and northern Italy 1348-1600. Canterbury: University of Kent, UP.

Petty, William. 1899. The political anatomy of Ireland. In The economic writings of Sir William Petty, Hrsg. Charles Henry Hull, 121-223. Cambridge: Cambridge University Press.

Petty, William. 1986. A treatise of Ireland. In The economic writings of Sir William Petty, Hrsg. Charles Henry Hull, 546-621. Cambridge: Cambridge University Press.

Prantl, Heribert. 2008. Der Terrorist als Gesetzgeber. München: Droemer.

Prins, Jan. 1996. Hobbes on light and vision. In The Cambridge companion to Hobbes, Hrsg. Tom Sorell, 129-156. Cambridge: Cambridge University Press.

Rosen, George. 1958. A history of public health. New York: MD Publications.

Salzmann, C. 1932. Masques portés par les Médecins en temps de Peste. Aesculape 22 (1): 5-14.

Sarasin, Philipp. 2005. Michel Foucault zur Einführung. Hamburg: Junius.

Sarasin, Philipp. 2005/2006. „War of the Worlds“. Der Terrorismus und das Pocken-Modell. Anmerkungen über Liberalität nach Foucault. In Lab. Jahrbuch für Künste und Apparate, Hrsg. Kunsthochschule für Medien Köln, 151-163. Köln: Verlag der Buchhandlung Walther König.

Schmitt, Carl. 1938. Der Leviathan in der Staatslehre des Thomas Hobbes. Sinn und Fehlschlag eines politischen Symbols. Hamburg: Hanseatische Verlagsanstalt. 
Schmitt, Carl. [1938]2003. Der Leviathan in der Staatslehre des Thomas Hobbes. Sinn und Fehlschlag eines politischen Symbols. Stuttgart: Klett-Cotta.

Siegert, Bernhard. 2006. Passagiere und Papiere. Schreibakte auf der Schwelle zwischen Spanien und Amerika. Paderborn: Wilhelm Fink.

Skinner, Quentin. 1996. Reason and rhetoric in the philosophy of Hobbes. Cambridge: Cambridge University Press.

Slack, Paul. 1985. The impact of plague in Tudor and Stuart England. London: Routledge.

Stollberg-Rilinger, Barbara. 1986. Der Staat als Maschine. Zur politischen Metaphorik des absoluten Fürstenstaats. Berlin: Duncker-Humblot.

Ulbricht, Otto. 2005. Angst und Angstbewältigung in den Zeiten der Pest 1500-1720. In Gotts verhengnis und seine straffe. Zur Geschichte der Seuchen in der Frühen Neuzeit, Hrsg. Petra Feuerstein, 101-112. Wiesbaden: Harrassowitz Verlag in Kommission.

Vaughan-Williams, Nick. 2009. Border politics. The limits of sovereign power. Edinburgh: Edinburgh University Press.

Von Müller, Achatz. 1984. Der venezianische Karneval. In Karneval in Venedig, Hrsg. Rolf D. Schwarz, 123-138. Dortmund: Harenberg.

Weihe, Richard. 2004. Die Paradoxie der Maske. Geschichte einer Form. München: Wilhelm Fink.

Windisch, Martin. 1997. Staatstheorie und Bildform bei Thomas Hobbes. Zeitsprünge. Forschungen zur Frühen Neuzeit. Zentrum zur Erforschung der Frühen Neuzeit 1 (1): 117-165.

Zimmer, Carl. 2004. Soul made flesh. The discovery of the brain - and how it changed the world. New York: Free Press. 